ophthalmitis. At least 13.5 per cent. of the blindness, in children of school age is due to heredity.

"The public should be educated regarding the potential danger to the eyes in cases of systemic disease involving either degenerative changes or nutrititional deficiencies, as well as in infectious diseases." Miss Kerby notes that 521 children in the schools for the blind during 1940-41 are there because of ophthalmia neonatorum, and 230 children are there because of syphilis pre-natally acquired. There is a folding table of the causes of blindness among pupils in the schools and day classes for the year in question, and other tables show the names of the schools with totals of pupils in each; distribution by amount of vision remaining; age distribution; and age at onset of blindness, all of which merit careful perusal.

Miss Kerby is to be congratulated on this excellent report.

\title{
LIGHT AND VISION
}

[Abstrict of an Address by SIR JOHN PARSONS to the Illuminating Engineering Society on May 11, 1943.

The Illuminating Engineering Society was founded by Mr. Leon Gaster in 1909 for the purpose of promoting "good lighting," i.e. lighting " adequate and suitable" for the given performance. "It was designed to represent all aspects of the subject, and its members include gas and electric light experts, physiologists, ophthalmologists, architects, and others. The writer is an original member, past president, and fellow of the Society.]

Ar the time of its inception there was little guidance in lighting matters from ophthalmologists and physiologists; but it must be remembered that information on the physical side was also vague. Although engineers were becoming accustomed to foot-candles the few instruments for measuring illumination were primitive and cumbersome. There were as yet few records of the illumination actually provided in different classes of buildings. At that time, too, the conception of brightness in terms of candles per square inch was little understood, and there were no quick and convenient means of measuring the brightness of sources.

In those days, as Sir Francis Goodenough has said (" Illuminating Engineer," Vol. xxv, p. 276, 1922), "Technicians were somewhat apt to devote themselves to the perfecting of their tools 
rather than the objects they served. For many years the chief task had been that of producing light economically and abundantly." One may compare this with the printers, whose object seemed to be to produce a beautiful page, with no regard for legibility. Since then there has been a marvellous advance in the efficiency of illuminants, so that undue stress has-been laid upon physical intensity. None the less in the first paper contributed to the Society on Glare (" Illuminating Engineer," Vol. iii, p. 99, 1910) I drew attention to the dangers of excessive light and the importance of the distribution of light in the field of vision.

Further, as Dr. Wright has reminded me, the average illuminating engineer is scared stiff of anything at all academic about the eye or the visual response; just as, in my own experience, the average medical student is scared stiff of mathematics and analytical optics. Being confronted with the chaos of metre-candles, foot-candles, equivalent foot-candles, lamberts, lumens; and photons, not to mention daylight factors and brills, may account for the apathy of ophthalmologists.

These opposed limitations in mental outlook are responsible for - many of the errors and shortcomings of both physicists and physiologists, and for the lack of liaison between them. As I have of ten pointed out physicists tacitly ignore the physiological basis of alt their measurements, hiding their heads under the convenient term " errors of experiment," and resorting to statistical methods to condone their ignorance. On the other hand there are many reasons into which I cannot enter now which militate against physiologists devoting themselves to research on the special senses; and psychologists who, ought to make these subjects their own prerogative, are nearly without exception ill equipped for the purpose. Nearly all the experiments which have been made by psychologists and many by physiologists are almost useless owing to the absence of accurate quantitative data. The experiments of von Kries and his pupils on colour vision and those by Lythgoe on peripheral vision, etc., are noteworthy exceptions.

The problems with which this Society is concerned are preeminently such as cannot be segregated into purely physical and physiological compartments. The physicist has been forced to open his eyes to physiological facts. An early example is the "deviations from Newton's law of colour mixtures," as König called them; which are due to the difference of scotopic from photopic vision. On the other hand, one has only to recall the namés of Newton, Thomas Young, Clerk Maxwell, Helmholtz, König, Abney, and many, others down to the present day to realise that we owe most of our knowledge to physicists.

The Illuminating Engineering Society was founded for a definitely utilitarian purpose-that of promoting " good lighting." 
Good lighting is sufficiently well defined in the Factory Lighting Committee's report as " adequate and suitable" for the given performance. One is almost startled to find that the problem is. primarily and fundamentally physiological, for adequacy and suitability depend upon the performer's capacity. Ophthalmologists knew that prolonged near work under low illumination and unsuitable conditions led to fatigue, " eyestrain," headaches, and bad health. Industrial fatigue is difficult to measure, though much valuable work was done by the Industrial Fatigue Board of the Medical Research Council, using output as thescriterion. The retina appears to be insusceptible to fatigue in ordinary conditions, and eyestrain is chiefly due to fatigue of the extra-ocular muscles; hence the importance of measuring convergence capacity and latent squints. Even more potent in causing eyestrain are uncorrected errors of refraction. It is only comparatively lately that examination of the eyesight of school children and the correction of their refractive errors have become compulsory; largely due to the pioneer work of Dr. Kerr and Mr. Bishop Harmanifor the London County Council. May we not hope that the sight of factory workers will be similarly catered for? A striking example of the value of the application of knowledge of this kind is the success of Mr. Weston's efforts to ameliorate the strain of very fine work by the use of suitable convex glasses combined with prisms.

Since good visual acuity is the sine quâ non of clear vision the effects of the quantity and quality of the illumination early attracted attention. Visual acuity is measured scientifically by the minimum visual angle subtended at the nodal point of the eye by two point sources necessary for resolution. So far as intensity is concerned we know now that the acuteness of vision becomes progressively better up to, and even beyond, 1,200 ft.c. Unfortunately, as Lythgoe has pointed out (Trans. I.E.S., Vol. i, p. 4, 1936), it was not sufficiently recognised in the early days that visual acuity is proportional to the logarithm of the illumination; so that on the linear scales then prevalent it appeared that there was little improvement above 3 or $4 \mathrm{ft} . c$. It is noteworthy that until the Council of British Ophthalmologists took the matter up in 1919 there was no standard fixed for the illumination of Test Types, and even so it was fixed at too. low $\mathrm{a}^{-}$level, which had to be increased in a subsequent report (1935).

Even scientifically disposed ophthalmologists are liable, like other scientists, to simplify their problems unduly, and consequently to attach undue importance to isolated details. Thus, little attention was paid in the above determinations to the surrounding illumination. It has since been found that this has a very marked effect. To get the best result the surrounding illumination should be only slightly less than that of the test object, book, or work. If 
it is much less visual acuity falls off after a maximum at about 40 e.f.c. illumination of the test object, and if it is quite dark the maximum is reached at less than 20 e.f.c.

The Society deserves to be congratulated upon the issue of the I.E.S. Code of Illuminations adequate and desirable for different classes of work. It is largely founded upon empirical data, and much research yet remains to be done; but that it filled a long felt need is shown by the hearty reception it has had.

Taking together the now justified predilection for higher illumination for reading and work, and the economic availability of greatly increased artificial illumination it is not surprising that people demand these higher illuminations, especially when we find that if the general illumination exceeds that of the test object visual acuity will be seriously diminished. Whether the satisfaction of this desire is being carried to extremes with deleterious results is a matter for future investigation; but the suggestion that the demand for high illumination is due to general deterioration of sight in the population is entirely unsupported by any valid evidence.

The effect of the surrounding illumination on visual acuity is one of the many characteristics of the eye as an optical instrument of precision which are so exasperating to the physicist. The retina is a part of the brain, and it is no surprise to the physiologist that there is interaction between the different parts. Much light has been thrown in recent years on this subject by the investigation of the electrical responses to stimuli by Adrian and Matthews, Hartline, Granit and others, and by the researches of Lythgoe and Tansley. It has long been known in the manifestations of simultaneous contrast. In its most blatant form excessive contrast plays a preponderating part in glare, as was indeed fully recognised in the earliest days of the Society. It was to the discussion on Glare, initiated by my paper at the first meeting that Prof. L. Weber of Kiel contributed recommendations which were substantially adopted by the Factory Lighting Committee and have even now been tittle modified. He advocated (1) a limit to brightness (about that of a candle flame) and (2) the location of sources outside the $30 \mathrm{deg}$. angle at the eye. But he also approached near to the root of the matter in suggesting that (3) the ratio of the brightness of the source to that of the surroundings should not exceed 100 to 1 -a value deduced from daylight conditions.

The earlier researches aimed at a satisfactory explanation of glare by Cobb and others were disappointing, and we owe it to Dr. Stiles that it has been put upon a more satisfactory basis, though I am sure he would be the first to admit that we have only touched the fringe of the subject. Recognising that glare is largely due to scattered light, i.e., light in the wrong place, he conceived the 
brilliant idea of translating its effects into those produced by flooding the whole area of the retina with uniformly distributed light; thereby enabling the results to be expressed in comparable quantitative terms.

We have at present no means of detecting glare directly in the physiological sense. We have therefore to fall back on indirect and empirical methods of controlling the circumstances which, it is conjectured, will give rise to glare; for example, by limiting the brightness of sources, or specifying for them positions outside the immediate range of vision. Possibly some simplification might be effected by some general rule specifying the desirable contrast or brightness ratio between object and surroundings, which would not only act as a safeguard against glare, but also ensure conditions favourable to the powers of perception of the eye. In this connection one must not forget glare due to specular reflection from polished material; nor the inconvenience and occasional danger of harsh shadows. The precise definition of shadow offers as many difficulties as the assessment of glare.

With regard to the quality of light our eyes are not only capable of adaptation to the enormous range from 0.00001 to $10,000 \mathrm{ft} . c$., but also to considerable variations of spectral constitution. Phylogenetically eyes have obviously been adapted-in the evolutionary sense of the word-to sunlight, but there is no evidence that artificial sources of so-called " white light," composed of practically continuous spectra, per se cause any harmful effects. These are generally due much more to the distribution of the light than to either quantity or quality. It is true that they nearly all, including the sun, emit ultra-violet radiation, but the atmosphere usually acts as an efficient filter in the case of the sun, and artificial sources. are enclosed in glass which is equally efficient. The deleterious effects of ultra-violet light in everyday life have been grossly exaggerated. Similarly, the craze for "daylight" lamps, apart from special colour-matching processes, etc., is quite unwarranted.

The effects of monochromatic light and of sources like mercury vapour lamps which emit discontinuous spectra, and of those with intermittent emission; especially when the cycles per second are few, are more debatable.

The phenomenal advance in the development of discharge lamps using fluorescent substances-an advance due largely to the G.E.C. Research Laboratory under the aegis of Mr. C. C. Paterson, F.R.S.-emphasises the importance of colour vision and its defects. The work of Guild and others on Colorimetry resulted in 1931 in the standardisation of colour mixture curves by the International Commission of Illumination-a fitting sequel to the standardisation of the equal energy luminosity curve in 1924 by the 
same body. Together they form perhaps the most noteworthy advance in photometry of recént date. Neither has the theoretical side been neglected. Dr. W. D. Wright's reinvestigation of the so-called sensation curves by more refined methods has corrected some discrepancies in the work of König and Abney; and by using the method of binocular matching, in which adaptation effects induced in one eye are measured by comparing vision in that eve with vision in the constantly dark-adapted other eye, he and others, e.g., Schouten and Ornstein, Pitt and Craik, have elicited new facts of theoretical and practical value.

Considering the great prevalence of colour blindness in different degrees - at least 5 per cent. of the male population are badly colourblind - it is remarkable how little this disability seems to affect everyday l.fe. Yet many of these are constantly engaged in delicate photometric observations. In most such tests difference in hue of the test objects is reduced to a minimum, so that the estimate is one of equality of brightness in two white lights. There is some evidence that pure brightness discrimination is actually better in the colourblind than in the normal-a fact which has been adduced as a factor in the excellence of the etchings of Méryon. I suppose that in the grosser forms of heterochromatic photometry the results are always controlled by the flicker method. We may hope that 1$) r$. Wright's researches will throw further light on the luminosity curves of the colour-blind. Even in colour matching, however, such colour-blinds as conform with the Trichromatic Theory would be expected to accept the normal matches. In fact, they do so, but, unfortunately, as Prof. William Watson pointed out, they. will also accept considerable deviations from the normal match.

Until the outbreak of the war and the initiation of the " blackout "illuminating engineers paid little attention to the peculiarities of vision under very low illuminations. Probably their first experience of these was due to anomalies in photometric observations caused by the Purkinje phenomenon. Previous to the " blackout " town dwellers had little experience of complete dark adaptation, and it is therefore not surprising that they should be found to be psychologically relatively night-blind compared with country dwellers. Attention was first seriously devoted to the subject when exhaustive researches-were made to improve street lighting and put it on a satisfactory scientific basis. The Factory Lighting Committee were faced with the problem of setting up standards for the illumination of passages in factories, non-working areas, dockyards, and so on. These were fixed as follows: $(a)$ over interior parts of a factory other than working areas, $0.1 \mathrm{ft}$.c. (1915), subsequently increased to $0.1 \mathrm{ft} . \mathrm{c}$. to $0.5 \mathrm{ft} . \mathrm{c}$. $(1938)$; (b) over open yards, etc., not less than $0.05 \mathrm{ft} . \mathrm{c}$, subsequently increased to form $0.05 \mathrm{ft} . \mathrm{c}$. to $0.1 \mathrm{ft} . \mathrm{c}$. In the present circumstances the conditions 
of vision under moonlight and starlight illumination are literally of vital importance to airmen and other combatants.

None of the standards hitherto set is based on really satisfactory scientific evidence, partly on account of the ignorance of physicists and illuminating engineers of such facts of the physiology of scotopic vision as are known, but much more because of the ignorance of the physiologists themselves. A vast amount of very valuable work has been done on night vision for the Services, especially the R.A.F., and, while it has been of immense practical utility, it has but served to reveal the extreme complexity of the subject.

Dark adaptation is characterised by a phenomenal increase (from about $0.00001 \mathrm{ft} . c$. to $10,000 \mathrm{ft} . c$.) in the sensitivity of the retina, which rises rapidly during the first ten or fifteen minutes and reaches a maximum in about three quarters of an hour. This rise in sensitivity shows some variation in the normal population, but there are pathological cases in which it is delayed and defective and may be very bad.

When a spectrum of low intensity is viewed by the dark adapted eye it appears as a colourless band, having a luminosity curve which is of the same general type as the photopic luminosity curve, but having its maximum at about $500 \mu \mu$ instead of $550 \mu \mu$. This shift of the luminosity curve accounts for the Purkinje phenomenon, in which the apparent brightness of the short wavelengths is much enhanced compared with the longer wave-lengths.

Visual acuity, even under photopic conditions, falls off very rapidly as the fixation point is shifted from the centre, i.e., direct vision to the periphery.

The fovea which is the site of highest discrimination or visual acuity, subtends a visual angle at the nodal point of the eye of $60^{\prime}$ to $70^{\prime}$; the macula is oval, with the long axis horizontal, subtending a visual angle of $4^{\circ}$ from above down and $12^{\circ}$ across. The deeper layers of the retina not only conduct impulses directly to the brain, but have intercommunications which permit of modifications in the responses of adjacent areas.

It is certain that the rods contain a photochemical substance which subserves the transformation of the physical stimulus into a nervous impulse. This substance, rhodopsin or visual purple, appears to be absent from the cones, where it is almost certainly replaced by a similar photochemical substance or substances. $V$ isual purple is bleached by light, and its absorption curve is identical with the scotopic luminosity curve, so that it is practically certain that it is the physiological basis of vision under very low illuminations. Dark adaptation is due to the accumulation of visual purple, and since rods are absent in the greater part of the macula, it might be expected that this area would be night-blind. 
That this is indeed the case was discovered long ago by astronomers, who found that a star of very low magnitude could be seen only by looking at it eccentrically, so that the image fell upon a parafoveal spot. The macula is not, however, wholly incapable of dark adaptation : such as it possesses is indeed slight, but rapid in development.

The researches of Lythgoe and Goodeve, and of Wald have thrown much light on the constitution and properties of visual purple. It has long been known that in certain cases of malnutrition, e.g., xerophthalmia, night blindness is a cardinal symptom. It is now known that the presence of vitamin $A$ is essential to the regeneration of rhodopsin. As a result a vast amount of work has been done in the hope of improving night vision by administering large doses of vitamin $\mathrm{A}$. The evidence seems to prove that this has little or no effect on normal subjects, but that if the defect is due to malnutrition the effects are striking.

König showed many years ago that if the effects of illumination on brightness discrimination are plotted on a logarithmic scale two straight lines of different slope result, the kink occurring at about 0.25 metre-candles $(0.023 \mathrm{ft} . \mathrm{c}$.). This has been confirmed by flicker photometry : and the same curves are obtained with the effects of illumination on visual acuity. There can be little doubt that, in accordance with the Duplicity 'Theory, the scotopic part of the curve is due to rod vision and the photopic to cone ( \pm rod) vision.

The transition from day vision to night vision therefore occurs when the illumination decreases to less than that provided by the full moon, about $0.023 \mathrm{ft} . c$. , night vision being fully established at illuminations of less than $0.01 \mathrm{ft}$.c. Above the upper limit photopic, foveal, cone vision will be in the ascendant; fixation will be good, and visual acuity good. Below the lower limit scotopic, rod vision will be used; fixation will be bad, parafoveal, and fluctuating owing to rapid fatigue of the rods, and visual acuity will be bad. At the intermediate levels fixation will be fluctuating, and it is likely that vision will be worse than might be expected owing to rivalry between the two systems.

But there are other factors which make the complexity of scotopic vision much greater. The chief are the size of the area stimulated and the contrast or brightness discrimination at these low levels of illumination. For visual angles up to about $40^{\prime}$ to $50^{\prime}$ Riccò's law holds good for central vision; i.e., at the threshold the product of the area into the light intensity is constant. The threshold luminous flux is about $0.2 \times 10^{-12}$ lumen for extra-foveal, and $8 \times 10^{-12}$ lumen for foveal vision. For larger areas the total luminous flux is no longer alone responsible for the threshold. According to Piper the product of the threshold intensity into the square root of the area is constant, but this is only valid for 
white light and objects subtending a visual angle up to $10^{\circ}$ (Henius, Fujita). Similar laws hold good for the detection of the orientation of Landoldt's broken ring (C), or an $E$; but this only shows that these are really tests of brightness discrimination, and are quite unsatisfactory tests of true visual acuity or form visionat any rate at low intensities of illumination.

Brightness discrimination depends upon the area even at normal levels of illumination. With a photometric field of $5^{\circ}$ the eye has to work with regions of the retina which differ in sensitivity, and more consistent results are obtained when the visual angle is reduced to $2 \frac{1}{2}^{\circ}$. At $1^{\circ}$ vision is foveal and purely photopic, and there is no kink in the logarithmic curve. The transition occurs at about 0.03 e.f.c., the order of brightness provided by the full moon, and is responsible for the low values of $A B / B$ which are obtained with large fields at intensities far below those at which discriminating capacity vanishes with smaller areas. It is important to remember that ${ }^{\Delta} \mathrm{B} / \mathrm{B}$ expresses only relative brightness difference in terms of stimulus values, and is only valid as an expression of physiological apparent brightness difference or true contrast over the limited range in which the Weber-Fechner law holds good. The difficult problem of brightness and contrast is discussed in an excellent paper by Hopkinson, Stevens, and Waldram (Trans. I.E.S., Vol. vi, p. 37, 1941). The discordant results obtained by different observers are largely due not only to the differences of size and shape of the test areas, but also to the size of the illuminated surrounds. When the test field is exposed for only a short period of time a higher percentage of contrast is needed to produce a just noticeable difference.

Hopkinson, Stevens, and Waldram point out that when the adaptation level is reduced the instantaneous visual range of appreciable brightness is not symmetrically disposed about the adaptation level, as is approximately the case at the normal levels. "The eye can then distinguish brightnesses greater than that to which it is adapted, but not much to lower brightnesses "-a fact of "great significance in street lighting and aeroplane spotting. It is also of considerable importance in the measurement of low values of illumination and brightness, a subject dealt with by Dr. Walsh (Trans. I.E.S., Vol. vi, p. 117, 1941). There is little reference to physiological conditions in this paper, except that in speaking of the Purkinje phenomenon the author says that " one can easily live in a fool's paradise," It seems to me that, in the present state of knowledge, the same remark applies to making measurements in which " the range introduced is 0.0005 to $0.3 \mathrm{ft} . c$., and the corresponding limit of error laid down is 20 per cent. + 0.0001 ft.c." (Specification for Portable Photometers (Visual Type), 230: 1935). Fortunately, Dr. Walsh realises that " the necessity 
for measuring apparent brightness, rather than brightness based on a physical scale, is perhaps the most striking of these new developments."

The lowest level at which consistent data as to the influence of contrast on visual acuity have been obtained is 1 ft.c., which. is much greater than that prevailing at night; even for this illumination for less than 10 per cent. contrast large increases of size have to be made. Riccò's and Piper's results indicate the decreased importance of contrast at low intensities. - Their results also show that in using field-glasses at night, with visual angles between $10^{\prime}$ and $2^{\circ}$, the size of the exit pupil, and therefore the amount of light gathered, is more important than the coefficient of magnification.

- [The speaker then reviewed some of the activities of the Society in the past : e.g.,. lighting of schools and libraries (1913), the lighting of factories and workshops, eyestrain in cinemas (1920), the I.E.S. Code of Illuminations].

The main functions of the Society have been well catered for in the past, and the formation of provincial Centres and Groups has thoroughly justified itself by arousing local interests and attracting attention to the needs of Yocal industries. But " these things ought ye to have done, and not to leave the other undone," the amenities of everyday life might be considerably improved if more detailed attention were paid to such problems as the distribution, quality, and aesthetic effects' of domestic lighting. The experience of the Factory Lighting Committee has shown that problems of this kind are not so simple as might be thought. Many of the inconveniences which we grumble at but put up with $\rightarrow$ rooms in which it is almost impossible to read or sew with comfort, bedrooms with half a dozen'mirrors in none of which it is possible to see to part one's hair or shave-might be eliminated or ameliorated if architects would take the trouble to learn from illuminating engineers and insist upon builders carrying out their instructions, such as putting in the lighting points where they ought to go, and not where the builder finds it most convenient for himself. The definition of the edges of steps and stairs and their lighting still seems to me to be unsatisfactory. Perhaps these and other similar problems might be referred to an Architectural Commiftee : but what I think is much more necessary is the establishment of a standing Physiological Committee. 